\title{
Clinical significance of detecting CSF-derived tumor cells in breast cancer patients with leptomeningeal metastasis
}

\author{
Xuelu Li ${ }^{1,2, *}$, Yuan Zhang ${ }^{1,3, *}$, Jinlei Ding ${ }^{1,4, *}$, Min Wang ${ }^{1,3}, \mathbf{N a ~ L i}^{1,5}$, Hui Yang ${ }^{1,5}$, Kainan \\ Wang ${ }^{1,5}$, Dandan Wang ${ }^{6}$, Peter Ping Lin ${ }^{6}$, Man Lí ${ }^{1,5}$, Zuowei Zhao ${ }^{1,2}$ and Pixu Liu ${ }^{1,3,7}$ \\ ${ }^{1}$ The Second Affiliated Hospital, Institute of Cancer Stem Cell, Dalian Medical University, Dalian, China \\ ${ }^{2}$ Department of Breast Surgery, The Second Affiliated Hospital, Dalian Medical University, Dalian, China \\ ${ }^{3}$ Institute of Cancer Stem Cell, Cancer Center, Dalian Medical University, Dalian, China \\ ${ }^{4}$ Cancer Institute, The Second Affiliated Hospital, Dalian Medical University, Dalian, China \\ ${ }^{5}$ Department of Oncology, The Second Affiliated Hospital, Dalian Medical University, Dalian, China \\ ${ }^{6}$ Cytelligen, San Diego, California, USA \\ ${ }^{7}$ College of Pharmacy, Dalian Medical University, Dalian, China \\ "These authors contributed equally to this work \\ Correspondence to: Pixu Liu, email: pixu_liu@dmu.edu.cn \\ Zuowei Zhao, email: dmuzhaozuowei@163.com \\ Man Li, email: dmuliman@163.com \\ Peter Ping Lin, email: plin@cytelligen.com
}

Keywords: breast cancer; leptomeningeal metastasis; cerebrospinal fluid-derived tumor cell; SE-i $\bullet F I S H$

Received: July 20, $2017 \quad$ Accepted: December 14, $2017 \quad$ Published: December 21, 2017

Copyright: Li et al. This is an open-access article distributed under the terms of the Creative Commons Attribution License 3.0 (CC BY 3.0), which permits unrestricted use, distribution, and reproduction in any medium, provided the original author and source are credited.

\section{ABSTRACT}

Despite marked advances in breast cancer therapy, breast cancer-associated leptomeningeal metastasis (LM), a particularly aggressive syndrome with multifocal seeding of the leptomeninges by tumor cells, still carries an abysmal prognosis. A major problem with breast cancer LM surveillance is the lack of an effective and sensitive means to track dynamic changes of the disease. Cytology detection of cerebrospinal fluid (CSF) is considered the gold standard for LM diagnosis but has a high falsenegative rate with a limited sensitivity. Here we applied subtraction enrichment and immunostaining-fluorescence in situ (SE-i•FISH) method, a technique previously used for isolating circulating tumor cells (CTCs) from the peripheral blood, to detect, enumerate, and track cerebrospinal fluid-derived tumor cells (CSFTCs) in CSF samples from 8 breast cancer patients. Comparing with cytology test, we found SE-i•FISH method can accurately and feasibly detect CSFTCs for the diagnosis of breast cancerassociated LM and monitor the disease progression. We also isolated and cultured CSFTCs from these cancer patients and performed genomic sequencing on CSFTCs of two patients. Genomic analysis of CSFTCs against corresponding archival primary breast tumors revealed clonal relationships with some ongoing evolution. Further drug sensitivity test on cultured CSFTCs based on genomic analysis data helped identify promising treatment options for the patient tested. Together, our results suggest that CSFTCs detection using SE-i•FISH platform could serve as a sensitive and accurate method to make the diagnosis and a promising approach to monitor tumor dynamics and treatment response for breast cancer-associated LM. 


\section{INTRODUCTION}

The cerebrospinal fluid (CSF) lies in between leptomeninges, which are the membranes lining the brain and spinal cord [1]. Leptomeningeal metastasis (LM), also called neoplastic meningitis and carcinomatous meningitis, is a particularly aggressive syndrome with very high mortality $[2,3,4]$. LM from breast cancer has been reported to occur in approximately $2 \%-5 \%$ of cases over the natural course of the disease $[1,2,3]$. With development of more sensitive diagnostic technology and better imaging modalities that allow for more accurate and earlier diagnosis, along with the improvement in effective targeted drugs that extend patients' lives, the incidence of LM is on the rise $[1,2,3]$. Management strategies available for breast cancer patients with LM are limited without published guidelines, only including whole brain radiation therapy (WBRT), surgery, stereotactic radiosurgery (SRS), intrathecal and systemic chemotherapy [2]. Treatment remains difficult and little is known about the particularly aggressive metastatic process. Therefore, it is urgent to develop a feasible and reliable method to help diagnose LM and monitor the treatment response of this type of breast cancer patients.

The best test for LM detection is to obtain CSF samples by performing a lumbar puncture and subsequently the CSF is examined for cancer cells using papanicolaou staining. Unfortunately, the sensitivity of cytology examination of LM is at approximately $50 \%$ and $85-90 \%$ for the first and the second puncture, respectively [2]. As the sensitivity and methods for detection of LM are relatively limited, we set out to establish a subtraction enrichment and immunostaining-fluorescence in situ hybridization (SE-i•FISH) platform for isolation and functional analysis of cerebrospinal fluid-derived tumor cells (CSFTCs) with the purpose to improve the diagnosis, reduce false negative results, and gain molecular understanding of this disease. The SE-i•FISH platform has been previously used for enrichment and identification of circulating tumor cells (CTCs) from blood, which is irrespective of the specific surface marker expression and cell size variation. The unbiased enrichment and identification of highly heterogeneous CTCs/CSFTCs lead to a higher tumor cell detection efficiency compared to the conventional CSF cytological examination.

In this study, we employed SE-i•FISH platform to detect and isolate CSFTCs from 8 breast cancer patients with LM, an approach by which we can enrich nonantibody perturbed native tumor cells suitable for primary cell culture and additional biological analyses. We found that enumeration of CSFTCs could help make a quick and accurate diagnosis and evaluate treatment response of LM. Furthermore, genomic and functional analyses of cultured
CSFTCs may help identify mutated drug targets and assist preclinical personalized drug sensitivity test.

\section{RESULTS}

\section{Patient characteristics}

Eight breast cancer patients have been diagnosed with leptomeningeal metastases (LM) (Table 1). The cerebrospinal fluid (CSF) samples were obtained from these patients at the initial diagnosis of LM and before intrathecal chemotherapy. Cerebrospinal fluid-derived tumor cells (CSFTCs) were observed in all patients by standard cytologic detection of malignant cells in CSF. Neurological symptoms, such as headache, vision changes and nausea, were present in eight patients with LM. Seven of eight cases (87.5\%) showed positive magnetic resonance imaging at the time of initial LM diagnosis. All patients had presented multi-organ metastases before diagnosis of LM, mainly including bone and visceral metastases. The median age at diagnosis of primary breast cancer was 37 years (range 27-51). The median interval between the diagnosis of primary breast tumor and LM was 4.5 years (range 0.5-9 years), and the median overall survival time after the diagnosis of LM was 10.5 months (range 0.8-13 months). Eight patients exhibited progressive disease and died thereafter. The causes of death were all cancer-related.

Besides the 'gold standard' cytologic detection, we also employed a recently reported SE-i $\bullet$ FISH platform to monitor the number of CSFTCs during intrathecal chemotherapy (Figure 1A) [5, 6]. The concentrations of CSFTCs in threes patients (NM01, NM04, NM07) whose CSFTCs tended to generate tumor clusters in culture were higher than those from patients whose CSFTCs did not generate tumor clusters (Table 1 and Figure 1). We observed that the doubling time of CSFTCs in vitro culture ranged from 10-21 days (Table 1).

\section{Dynamic monitoring of CSFTCs}

To evaluate the feasibility of applying the SE-i•FISH platform to monitor treatment response, we retrospectively examined if the number of CSFTCs correlated positively with the intracranial pressure and neurological symptoms of the same patients. We found that there was a remarkable concordance between the number of CSFTCs and the aforementioned clinical markers (Figures 1A, 2D; Supplementary Tables 1,2). However, there was no such correlation between sensitivity to treatment response and the routine CSF cytology that only detected whether cancer cells were existent. These findings indicated that CSFTCs detection by the SE-i॰FISH platform might offer breast cancer LM patients a good opportunity to monitor the disease progression. 
Table 1: Summary of breast cancer patients with leptomeningel metastases

\begin{tabular}{|c|c|c|c|c|c|c|c|c|c|}
\hline \multirow[t]{2}{*}{ No. } & \multirow{2}{*}{$\begin{array}{c}\text { Age } \\
(P M T)\end{array}$} & \multicolumn{4}{|c|}{$\begin{array}{c}\text { Molecular classification of } \\
\text { PMT }\end{array}$} & \multirow{2}{*}{$\begin{array}{l}\text { Age } \\
(L M)\end{array}$} & \multirow{2}{*}{$\begin{array}{c}\text { Survival } \\
\text { Time } \\
\text { (LM to Death) }\end{array}$} & \multirow{2}{*}{$\begin{array}{l}\text { CSFTC count } \\
\text { per } 1 \mathrm{ml}\end{array}$} & \multirow[t]{2}{*}{$\begin{array}{c}\text { Time from } \\
\text { single cell to cluster }\end{array}$} \\
\hline & & $E R$ & $P R$ & HER2 & Ki67 & & & & \\
\hline NM01 & 27 & - & - & - & $30 \%$ & 28 & 9 months & 195 & 21 days \\
\hline NM02 & 34 & - & - & - & $50 \%$ & 43 & 1 year & - & No cluster \\
\hline NM03 & 40 & $50 \%$ & - & - & $30 \%$ & 47 & 20 days & 67 & No cluster \\
\hline NM04 & 46 & - & - & - & $80 \%$ & 48 & 6 months & 328 & 12 days \\
\hline NM05 & 33 & - & - & - & $50 \%$ & 39 & 8 months & - & No cluster \\
\hline NM06 & 43 & - & - & - & $40 \%$ & 49 & 11 months & 122 & No cluster \\
\hline NM07 & 51 & $<1 \%$ & $<1 \%$ & - & $40 \%$ & 51 & 1 year & 218 & 10 days \\
\hline NM08 & 32 & $70 \%$ & $50 \%$ & - & $20 \%$ & 35 & 13 months & - & No cluster \\
\hline
\end{tabular}

Note: PMT, primary tumor. LM, leptomeningel metastasis. '-', the data is not available.

\section{CSFTCs enriched by SE-i॰FISH platform can be cultured in vitro}

We next enriched tumor cells (subtraction enrichment, as reported in Materials \& Methods) from the chemotherapy-naive CSF to investigate whether CSFTCs could proliferate in vitro. We cultured CSFTCs in a medium without serum under suspension culture conditions so as to inhibit cell differentiation and maintain tumor cells at an undifferentiated state $[7,8,9]$. The CSFTCs from case NM01, NM04 and NM07 could be expanded and grew in vitro for at least seven weeks, but they gradually underwent senescence after that period (Figure 1B). However, attempts of CSFTCs ex vivo culture from the other patients were unsuccessful.

To identify potential actionable mutations and the origin of CSFTCs, we performed next generation sequencing on DNA extracted from CSFTCs and the matched primary breast tumors. For case NM01 and NM04, the genomic DNA exhibited sufficiently high purity, but the DNA libraries of primary tumor from case NM07 were not of sufficient quality. Therefore, the exome sequencing was only performed on NM01 and NM04. In case NM04, we identified non-synonymous SNVs of NOTCH2 and NUTM2A genes shared in both CSFTCs and the matched primary tumor and some private gene mutations either in CSFTCs or in the matched primary tumor, suggesting the common origin of the primary tumor and CSFTCs and ongoing clonal evolution during disease progression (Figures 1C, 4A; Supplementary Tables 3, 4). We also obtained similar findings in case NM01 that we will present later in details. Taken together, our results demonstrated that the SE-i•FISH platform could be used to isolate and culture CSFTCs and assist the molecular profiling of breast cancer associated LM.

\section{SE-i•FISH platform used in a typical patient}

Here we described the case of a 27-year-old female (NM01) who underwent a right modified radical mastectomy and then received a series of adjuvant therapies (Figure 2A). In December 2015, the postoperative chemotherapy had to be stopped for this patient due to excruciating headache and vomits, indicative of intracranial hypertension and likely leptomeningeal metastasis (Figure 2B). Initial MRI examination did not show leptomeningeal metastases that were diagnosed by CSF cytology (Figure 2C). The levels of tumor biomarkers (CA125, CA153 and CA199) in blood were within the normal range throughout the whole course of treatment, which was in discordance with the patient's condition. CSF cytology test was positive but it only detected whether cancer cells were existent without providing information about the dynamic change of tumor cells in the CSF. To this end, we monitored the number of CSFTCs and circulating tumor cells (CTCs) in blood during intrathecal chemotherapies using SE-i•FISH platform (Figures $2 \mathrm{~B}, 2 \mathrm{D}$ and $3 \mathrm{~A}$ ). Retrospectively, we found there was a correlation between the number of CSFTCs and clinical symptoms: when the patient presented with headache alleviation at the third intrathecal chemotherapy, we detected the number of CSFTCs as low as 12 per ml of cerebrospinal fluid (Figure 2D, Supplementary Table 1); when the headache of this patient became worse at the fifth intrathecal chemotherapy, we found the number of CSFTCs increased to as many as $95 \mathrm{per} \mathrm{ml}$. As there was no intravenous chemotherapy given in the course of intrathecal chemotherapy, the number of CTCs in blood increased gradually during this treatment period (Figure 2D). Our findings indicated that this method could dynamically monitor clinical response of the patient to intracranial chemotherapy and progression of the disease.

We next expanded CSFTCs from case NM01 in the special non-serum medium for 21 days and performed exome sequencing on CSFTCs and the matching primary breast tumor (Figure 3B, 3C). In addition, we carried out the drug sensitivity assay of these cultured CSFTCs (Figure 3D). Interestingly, we observed the morphological changes of CSFTCs after 52 days culture, some of which were small size cells (Diameter $\approx 3-4 \mu \mathrm{m}$ ), when comparing 


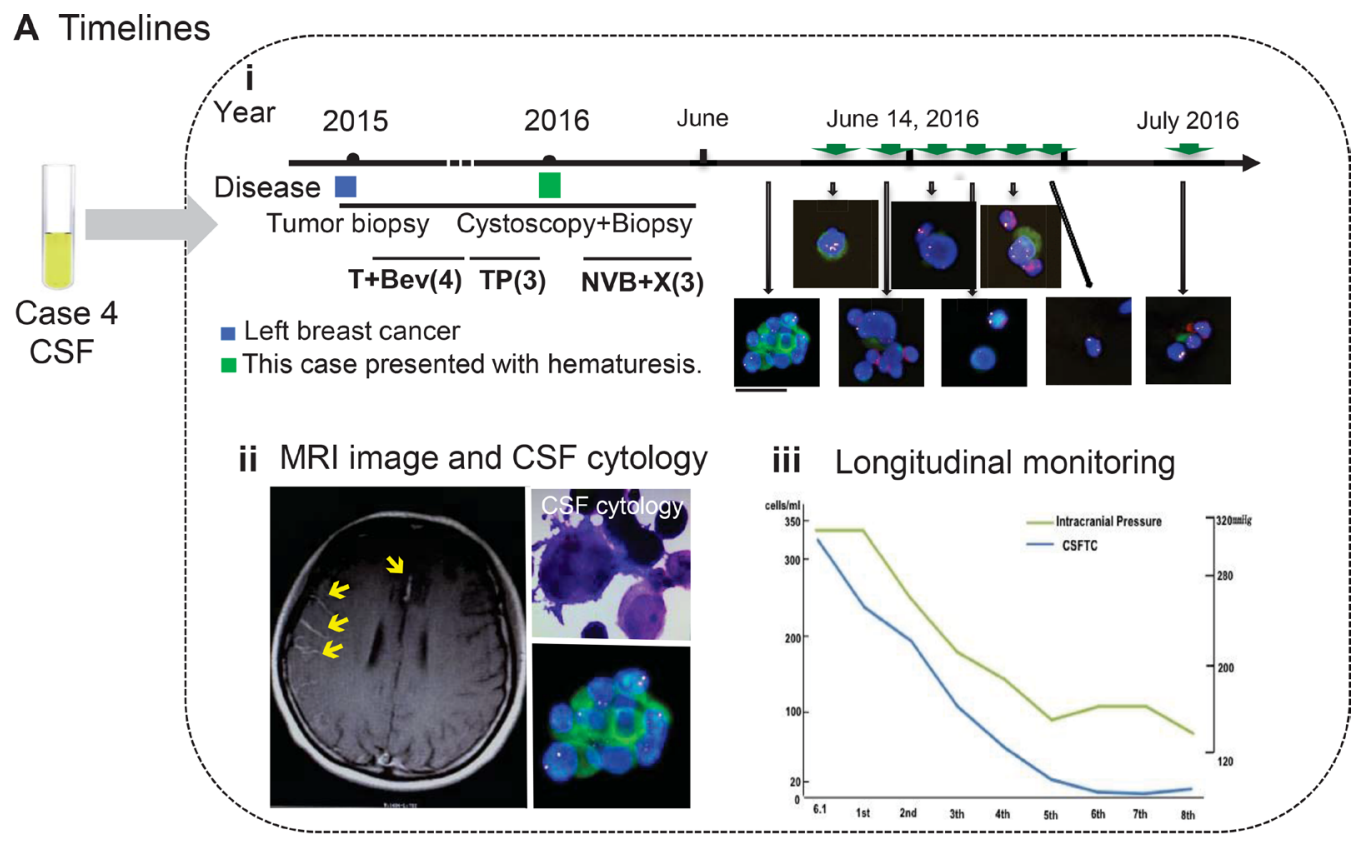

B Ex vivo culture of CSFTCs
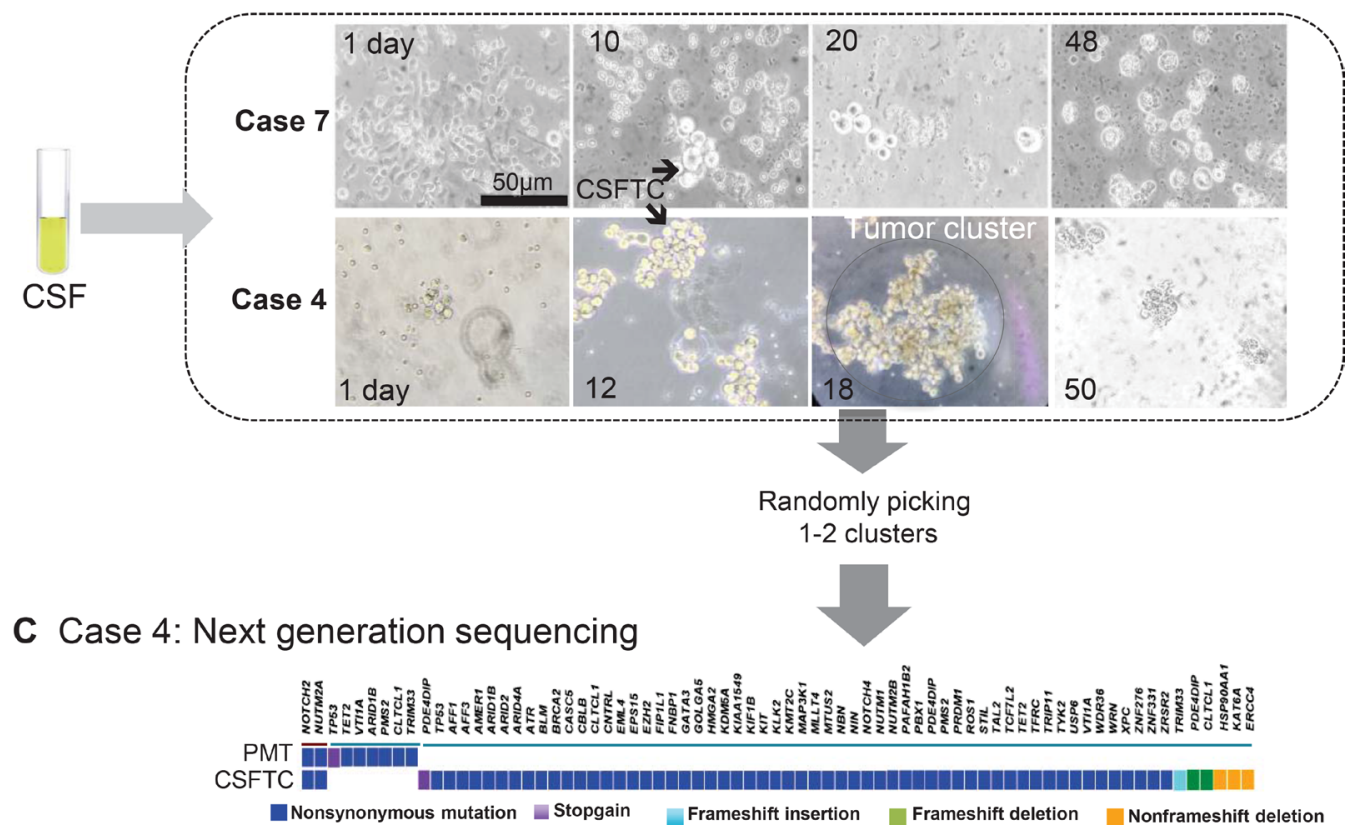

Figure 1: Overview of SE-i•FISH platform for dynamic monitoring and ex vivo culture of CSFTCs. (A) The treatment timelines received by the patient (NM04). (i) From 2015 until 2016, the patient received four cycles of adjuvant T+Bev (Paclitaxel/ Bevacizumab) and three cycles of TP (Paclitaxel/DDP) chemotherapy. In January 2016, the patient presented with hematuresis. Following urethrocystoscopy biopsy, the patient received three cycles of NVB+X (Vinorelbine/Capecitabine). The treatment had to stop as she presented with excruciating headache and vomiting. On the basis of the diagnosis of leptomeningeal metastases by MRI and CSF cytology, the patient received regularly intrathecal methotrexate and cytarabine. Green arrows mean intrathecal chemotherapies. The immunofluorescent staining for cytokeratin (CK, green), chromosome 8 (Yellow), CD45 (Red), and nuclei (DAPI, blue). (ii) Representative images of MRI and CSF cytology. (Upper right panel) Light microscopic imaging with Papanicolaou staining. (iii) The number of CSFTCs and the intracranial pressure are shown in the right panel. (B) Ex vivo culture of CSFTCs. 5-10 ml CSF were obtained from patients and then enriched. CSFTCs were cultured in a medium without serum under suspension culture conditions. The CSFTCs of NM04 and NM07 could be expanded in short term as indicated. Scale bar, $50 \mu \mathrm{m}$. (C) At Day 18, the tumor clusters of case NM04 were randomly picked. The exome sequencing was performed on DNA extracted from CSFTCs and the paired primary tumor. The distribution of somatic mutations detected by exome sequencing is presented in a heat map. 
with those cultured in the medium with $10 \%$ FBS (Figure 3E, 3F). A recent study showed that the existence of small sized tumor cells in the blood was correlated well with tumor metastatic potential [16]. We further characterized these small sized tumor cells from three aspects (Figure 3F): (1) FISH (chromosome 8) analysis indicated that these cell were human origin; (2) electron microscope observation showed the existence of organelles within the cells and the size of cells (about 3-4 $\mu \mathrm{m}$ ); (3) 3D culture assay revealed that these cells proliferated in a 3D condition (Supplementary Figure 1), proving their ability of growth in vitro.

\section{Drug sensitivity test on CSFTCs as a potential predictive tool in selection of treatment regimen}

We further characterized CSFTCs of case NM01 at the genome level by performing exome sequencing on CSFTCs and the paired primary tumor to look for actionable mutations that can be targeted. We identified

A

\begin{tabular}{|c|c|c|c|c|c|}
\hline 2014 & $\begin{array}{c}\text { June } \\
\perp\end{array}$ & $\perp$ & $\perp$ & June & December 27, 2015 \\
\hline \multicolumn{6}{|l|}{ Disease } \\
\hline \multirow{4}{*}{ Treatment } & \multicolumn{3}{|c|}{ Right modified radical mastectomy } & \multirow{2}{*}{\multicolumn{2}{|c|}{ 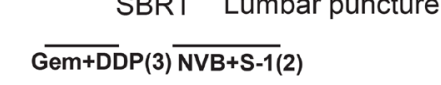 }} \\
\hline & & TAC(6) & Radiotherapy & & \\
\hline & Breast cancer (Right) & \multicolumn{4}{|c|}{ Left axillary lymph node metastasis, Lung metastasis, Bone metastasis } \\
\hline & Brain Metastasis & \multicolumn{4}{|c|}{ Leptomeningeal Metastasis } \\
\hline
\end{tabular}

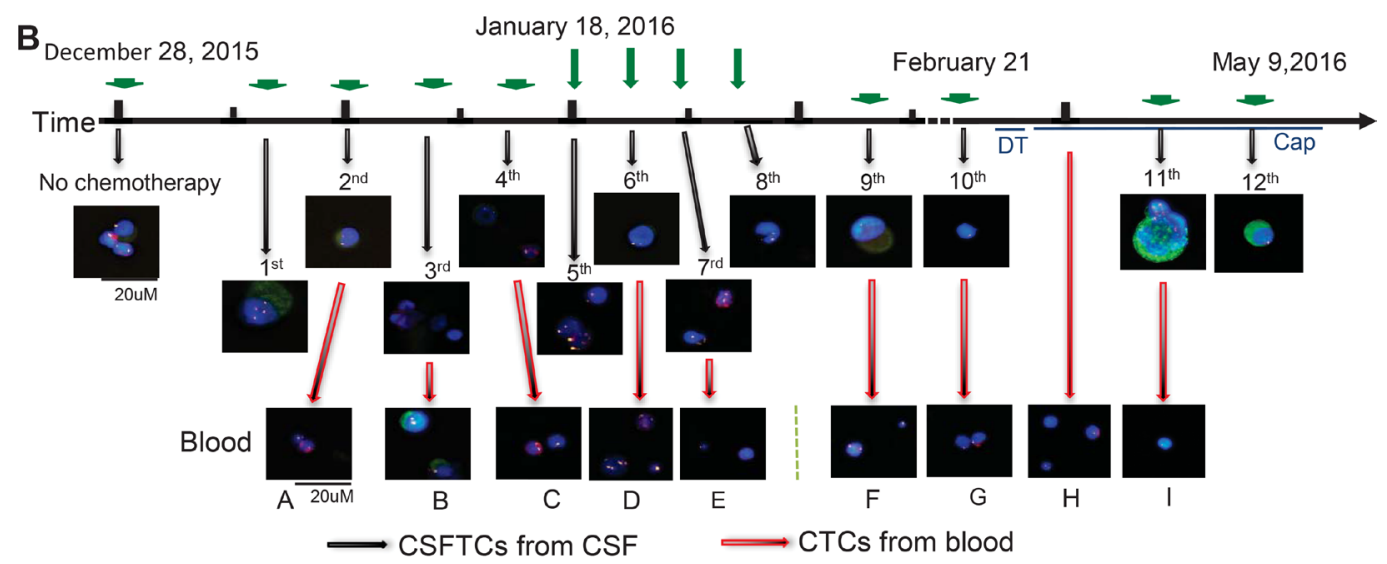

C Case1 Diffuse, non-adherent type

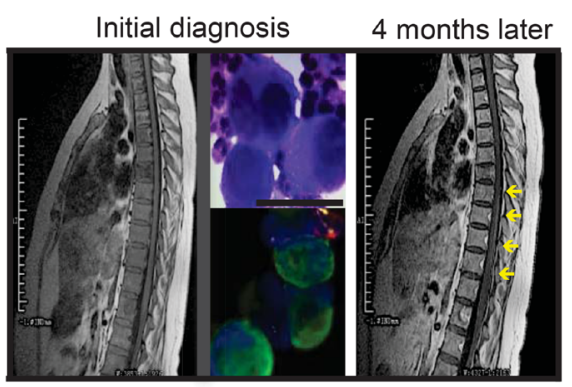

D Longitudinal monitoring

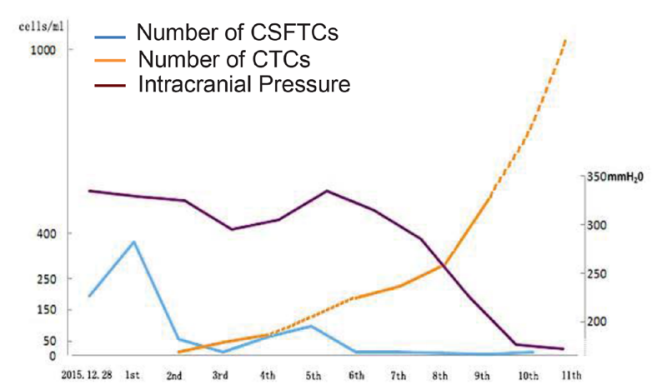

Figure 2: Treatment timelines and longitudinal monitoring of CSFTCs. (A, B) The treatment timelines received by the patient (NM01). From 2014 until 2015, the patient received six cycles of postoperative adjuvant TAC (Taxotere/Doxorubicin/Cyclophosphamide) chemotherapy, radiotherapy and three cycles of Gem+DDP (Gemcitabine/Cisplatin). In August 2015, brain metastases were detected and the patient underwent SBRT (Stereotactic body radiotherapy). Three cycles of NVB+S-1 (Vinorelbine/Tegafur) were given to the patient and the treatment had to stop as she presented with excruciating headache and vomiting. Leptomeningeal metastases were diagnosed by CSF cytology using Papanicolaou staining (C). In 2016, she received regularly intrathecal chemotherapy (Methotrexate/Cytarabine). Representative images of CSFTCs and CTCs are also shown. Green arrows mean intrathecal chemotherapies. Red arrows mean that we simultaneously monitored CTCs in blood. The immunofluorescent staining for cytokeratin (CK, green), chromosome 8 (Yellow), CD45 (Red), and nuclei (DAPI, blue). (C) Initial MRI examination did not show leptomeningeal metastases (left), whereas it appeared after 4 months. The yellow arrowheads are pointing to the leptomeningeal metastases (right). Dynamic changes of intracranial pressures (purple), CSFTC (blue) and CTC (yellow) counts as indicated in (D). 
somatic mutations in 61 genes with the primary tumor and CSFTCs carrying 30 private mutations respectively; one SNV of TP53 was shared by both the primary tumor and CSFTCs, suggesting heterogeneity and ongoing clonal evolution of the tumor cells examined in this case (Figure 4A, Supplementary Table 4).
We then tested the drug sensitivity of cultured CSFTCs of case NM01 to several most commonly clinically used chemotherapy drugs (cisplatin, paclitaxel, doxorubicin) and drugs for targeted inhibition (Figure 4B, Supplementary Table 5). It had been reported that breast cancer cells with p53 mutations exhibited impaired

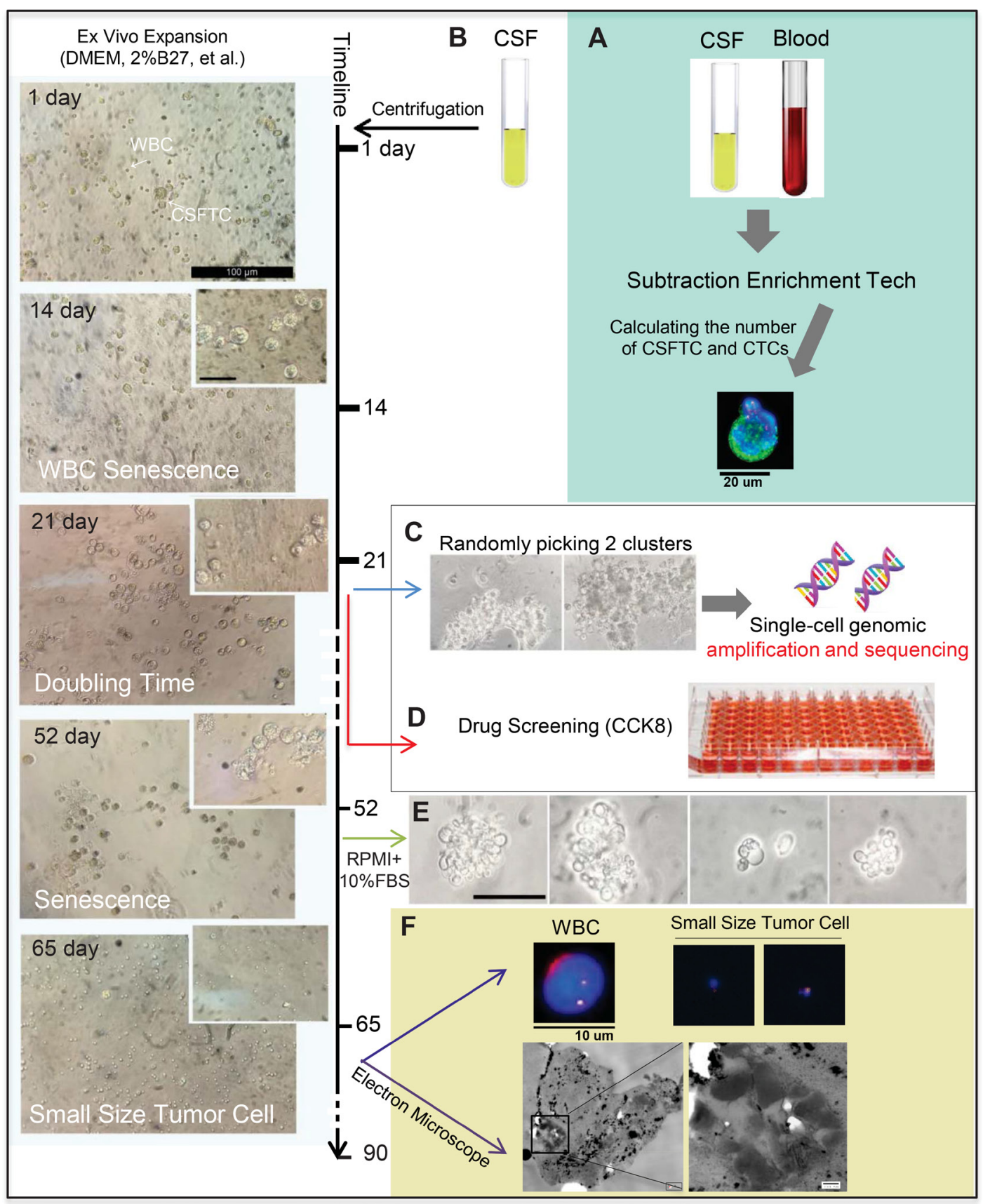

Figure 3: Ex vivo expansion of CSFTCs from case NM01. The left panel showed representative images of ex vivo culture of CSFTCs. The CSFTCs could be expanded for more than seven weeks, but after 90 days they gradually underwent senescence. (A) The number of CTCs in blood and CSFTCs were recorded by the SE-i $\bullet$ FISH platform. (B) Cerebrospinal fluid was collected and centrifuged. Subsequently, CSFTCs were enriched by subtraction enrichment (SE) technology. (C) At Day 21, cultures appeared tumor clusters, and then two tumor clusters were picked for single-cell genomic amplification and exome sequencing. (D) Ex vivo drug sensitivity test was assessed by CCK8 assay. (E) The morphological changes of CSFTCs were observed, when the special non-serum medium was switched to the medium with 10\% FBS. At Day 65, we observed the small size cells. Representative images of immunofluorescence (up) and scanning electron microscope (down) are shown in (F). 
DNA damage response and increased sensitivity to poly-ADP ribose polymerase (PARP) inhibitors [10]; in addition, INPP4B was proposed as a tumor suppressor to modulate PI3K signaling and lapatinib was a tyrosine kinase inhibitor that could prevent triple-negative breast cancer metastasis to the brain $[11,12,13]$. Based on these previous studies and our sequencing data, we chose to use PARP inhibitor Olaparib, PI3K inhibitor BKM120, tyrosine kinase inhibitor lapatinib. Several studies reported that Palbociclib could overcome CDK6 mutations (D163G, H100L) [14, 15]. We proposed CDK4/6 inhibitor palbociclib that would target CDK6 gene mutation (V77G) carried by CSFTCs in this case. Of all the drugs tested, selective CDK4/6 inhibitor palbociclib displayed the best anticancer effect, while the others were ineffective or just showed modest cytotoxic effects on CSFTCs viability test (Supplementary Table 5), suggesting that target inhibition of CDK6 in this patient would be an effective treatment option against CSFTCs (Figure 4). Together, our data indicated that ex vivo culture of CSFTCs may serve as a predictive model for assessing potential clinical therapeutic paradigms.

\section{DISCUSSION}

Here we describe an effective method for monitoring and ex vivo expansion of CSFTCs from breast cancer patients with leptomeningeal metastases. We found that there was a remarkable concordance between the number of CSFTCs and the aforementioned clinical markers such as intracranial pressure, the result of CSF cytology and clinical symptoms. On the other hand, the exome sequencing on CSFTCs identified potential actionable mutations that could be used to assist drug sensitivity test for each individual patient. All these data supported the notion that the SE-i•FISH platform could be applied to detect tumor cells in the CSF and more importantly, monitor the disease progression and treatment response.
Although CSF cytology is the current gold standard for LM diagnosis, this examination is notoriously insensitive $[1,2,16]$. Our results showed that the number of CSFTCs was concordant with headache alleviation and the dynamic changes of intracranial pressure. Lee et al. also reported the similar observation that changes in CSFTC counts predicted treatment response in seven breast cancer patients with leptomeningeal metastases [17].

The integrated SET-iFISH platform has been applied to detect and characterize CTCs in gastric, lung, and esophageal cancer patients $[5,6]$. In this study, we applied the SE-i•FISH platform for CSFTC enrichment on CSF samples from 8 breast cancer patients with leptomeningeal metastasis and cultured all of the CD45 negative remaining cells in non-adherent culture conditions. Only three lines of CSFTCs, namely NM01, NM03 and NM07, could be expanded ex vivo with relatively high concentration of tumor cells in the cerebral spinal fluid (>195 per ml), suggesting a requirement for certain tumor cell concentration for successful ex vivo culture of CSFTCs. For the specific CSFTCs from case NM01, we switched from the special non-serum medium to the medium with $10 \%$ FBS after seven weeks of cell culture and observed the morphological changes of CSFTCs, which were the small size cells. In addition, we also independently isolated small size tumor cells from blood and CSF samples of this patient at different time points. Our findings were consistent with the study from Chen et al. who reported that small nuclear CTCs were correlated with the tumor metastases [16, 18]. However, the clinicopathological features and molecular characteristics of these small size tumor cells remains to be identified.

The next generation sequencing identified the shared non-synonymous SNVs in both CSFTCs and the matched primary tumor, suggesting the common origin of these tumor cells. In contrast, we found some private gene mutations either in CSFTCs or in the matched primary tumor. The heterogeneous patterns of these genetic

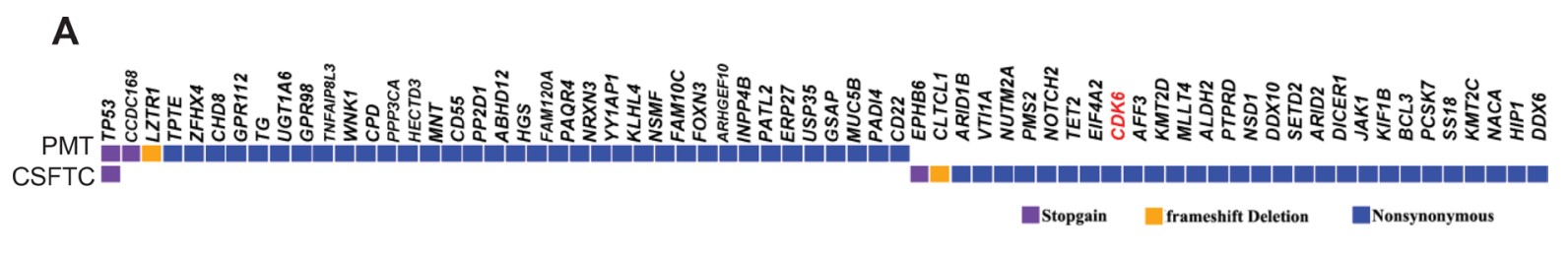

B

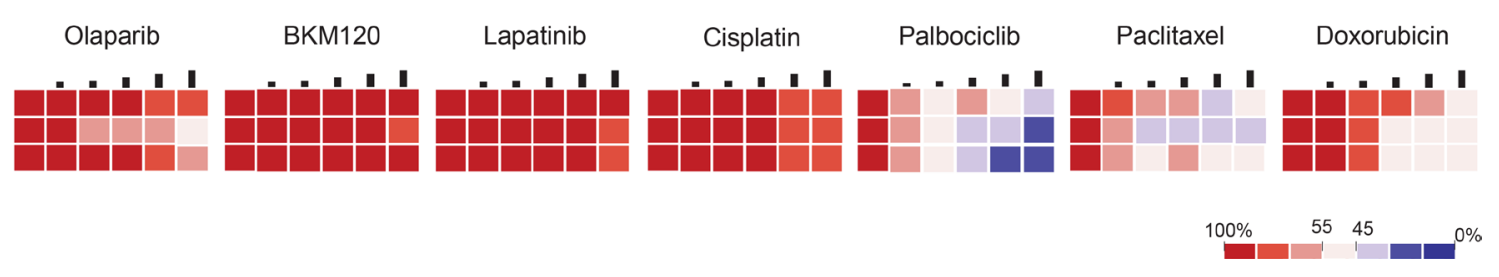

Figure 4: Drug sensitivity on CSFTCs as a potential predictive tool for treatment response. (A) The distribution of somatic mutations detected by exome sequencing was presented in a heat map. (B) Heatmaps represent cell viability after treatment of selected anticancer drugs. Each concentration represents a 2-fold increase from the previous dose, with each concentration tested in triplicate. 
mutations from primary tumor and CSFTCs suggested the ongoing clonal evolution during disease progression. In addition, drug sensitivity test and mutation analysis may help identify effective therapeutic strategies for each individual patient $[7,8,9,19,20,21]$. We noted that selective CDK4/6 inhibitor palbociclib exhibited the best anticancer effect with reference of the CDK6 gene mutation (V77G) carried by CSFTCs in case NM01. Our preclinical assessment of therapeutic options provides a potential effective treatment regimen based on the ex vivo drug sensitivity assay in combination with mutation analysis of this patient, a practice which needs to be validated in subsequent clinical treatment.

Together, CSFTC detection using SE-i•FISH platform provides a new approach to measure CSFTC levels in a dynamic manner. Changes in the CSFTC counts correlate well with LM disease state and therefore could serve as a predictor to monitor disease progression and treatment response of LM patients. In addition, the isolation and ex vivo culture of CSFTCs will facilitate molecular characterization of CSFTCs and their subtypes, as well as understanding the pathological nature of LM. The present study suggests that this platform may be commonly used in treating cancer patients with LM in the future. Further comprehensive profiling of CSFTCs at genomic, transcriptomic or proteomic levels in combination with functional studies of these tumor cells will help guide diagnosis, design of treatment regimes, drug response and prognosis for breast cancer LM patients.

\section{MATERIALS AND METHODS}

\section{Patients and samples}

Clinical samples were obtained from patients with breast cancer who were diagnosed with leptomeningeal metastases and were treated at the Second Hospital of Dalian Medical University between January 2013 and December 2016 (Table 1). T1 weighted enhancement magnetic resonance imaging (MRI) was the standard diagnostic procedure for patients with leptomeningeal metastases. Meningeal metastasis was also confirmed by cerebrospinal fluid (CSF) cytology. CSF samples were centrifuged. Subsequently, the smear was made by direct application of slide. Before the slide dried, it was fixed and stained according to Papanicolaou staining instruction. Eight patients provided their written informed consent. The study was approved by the Second Hospital of Dalian Medical University, and was performed according to the Declaration of Helsinki.

\section{Enumeration of CSFTCs and CTCs in blood}

Experiment was performed according to the product manufacture's instructions (Cytelligen, San Diego, CA, USA). Briefly, the peripheral blood and cerebrospinal fluid were collected into Cytelligen tubes containing ACD anti-coagulant (Becton Dickinson, Franklin Lakes, NJ, USA) and centrifuged at $450 \times \mathrm{g}$ for $5 \mathrm{~min}$. Supernatant was collected and incubated with anti-WBC (CD45) and endothelial cell immunomagnetic beads at room temperature for $20 \mathrm{~min}$ with gentle shaking. Subsequently, solution was subjected to magnetic separation. Beadfree solution was spun at $500 \times \mathrm{g}$ for $2 \mathrm{~min}$. The sedimented cells containing rare cells were mixed with $100 \mu \mathrm{l}$ cell fixative, and applied onto the formatted and coated slides for subsequent iFISH analysis. Air dried samples were suitable for subsequent analyses, including immunofluorescent staining (CK18, DAPI and CD45) and immunostaining-fluorescence in situ hybridization (centromere 8$)(\mathrm{i} \bullet \mathrm{FISH})$. Images of cells were visualized with a fluorescence microscope (Olympus, Tokyo, Japan). CSFTCs were identified as DAPI+/CD45-/CK+ or DAPI+/CD45-/CK- with aneuploidy or polyploidy of chromosome 8 .

\section{Cell culture}

Subtraction enrichment (SE) was performed according to the product manufacture's instruction (Cytelligen, San Diego, CA, USA). Enriched tumor cells were grown in 24well low-attachment plates with medium consisting of RPMI1640 medium (Gibco, Carlsbad, CA) supplemented with EGF (20 ng/ml), basic FGF (20 ng/ml), B27 (2\%), Insulin $(5 \mathrm{mg} / \mathrm{ml})$, Hydrocortisone $(0.5 \mathrm{mg} / \mathrm{ml})$ and Antibioticantimycotic (1\%) (Life Technologies) [7, 8, 9]. Culture medium was changed every 4 days under microscopic monitoring of CSFTCs. 3D cell culture experiments were performed as described previously [22]. Briefly, cancer cells were seeded on 96-well plates coated with $100 \mu$ l Matrigel (BD Biosciences). Cells were grown in the above-described RPMI-1640 medium supplemented with 2\% Matrigel. Cells were incubated at $37^{\circ} \mathrm{C}$ in a $5 \% \mathrm{CO}_{2}$-containing atmosphere.

\section{Next generation sequencing analysis}

The FFPE primary tumor tissues and normal oral epithelial cells were disaggregated, and gDNAs were isolated using QiaAmp FFPE DNA Mini kit and QiaAmp DNA Mini kit (Qiagen). The extracted genomic DNA was sequenced on the Illumina Hiseq2000 platform. To remove artifacts likely caused by cytosine deamination due to fixation/long-term storage of FFPE samples [23] and the low tumor cell content of samples, the following filtering criterion was applied: somatic mutations were called when supported by at least five reads, representing at least $10 \%$ of the total reads.

CSFTCs were disaggregated. Subsequently, singlecell genomic amplification was performed using REPLI-g Single Cell Kit (Qiagen). The target capture was performed using a custom Agilent SureSelect assay for 576 cancerrelated genes targeting an average coverage of $500 \times$ for 
the panel (Gentalker, China). To remove the presence of a low tumor cell content of samples, the following filtering criterion was applied: somatic mutations were called when supported by at least five reads, representing at least 5\% of the total reads.

\section{Drug sensitivity assay}

Cells were seeded one day prior to treatment in 96-well plates at 2000 cells per well. Each drug was added at 5 different concentrations. Cell viability was tested after 4 days of drug exposure using CCK-8 assay according to the product manufacturer's instructions (Dojindo Molecular Technologies), and was normalized to corresponding vehicle controls.

\section{Scanning electron microscopy}

Small size tumor cells were collected and centrifuged at $1050 \times \mathrm{g}$ for $5 \mathrm{~min}$. These cells were embedded into $2.5 \%$ agar and fixed in glutaraldehyde. The cells were observed with TEM at $120 \mathrm{KV}$ using ATM Camera System.

\section{Ethical approval}

The study was approved by the Second Hospital of Dalian Medical University, and conducted in accordance with the Declaration of Helsinki.

\section{Author contributions}

Pixu Liu, Zuowei Zhao, Man Li and Peter Ping Lin designed the study and revised the manuscript. Xuelu Li, Yuan Zhang and Jinlei Ding were responsible for manuscript drafting. Min Wang, Na Li, Hui Yang, Kainan Wang collected patient data. Xuelu Li, Yuan Zhang and Jinlei Ding were responsible for statistical analysis. Data analysis and interpretation: Pixu Liu, Zuowei Zhao and Man Li.

\section{CONFLICTS OF INTEREST}

The authors have declared no conflicts of interest.

\section{FUNDING}

This work was supported by the National Natural Science Foundation of China (No. 81071127, No. 81471751 and No. 81673762 to Z. Zhao; No. 81372853 and No. 81572586 to P. Liu; No. 81650018 to M. Li) and Provincial Natural Science Foundation of Liaoning (No. 2014023025 to M. Li; No. 2014023002 to P. Liu), Liaoning Provincial Climbing Scholars Supporting Program of China (2012 to P. Liu).

\section{REFERENCES}

1. Corbin ZA, Nagpal S. Leptomeningeal Metastases. JAMA Oncol. 2016; 2:839.

2. Mack F, Baumert BG, Schäfer N, Hattingen E, Scheffler B, Herrlinger U, Glas M. Therapy of leptomeningeal metastasis in solid tumors. Cancer Treat Rev. 2016; 43:83-91.

3. Magbanua MJ, Melisko M, Roy R, Sosa EV, Hauranieh L, Kablanian A, Eisenbud LE, Ryazantsev A, Au A, Scott JH, Park JW. Molecular Profiling of Tumor Cells in Cerebrospinal Fluid and Matched Primary Tumors from Metastatic Breast Cancer Patients with Leptomeningeal Carcinomatosis. Cancer Res. 2013; 73:7134-7143.

4. Gauthier H, Guilhaume MN, Bidard FC, Pierga JY, Girre V, Cottu PH, Laurence V, Livartowski A, Mignot L, Diéras V. Survival of breast cancer patients with meningeal carcinomatosis. Annals of Oncology. 2010; 21: 2183-2187.

5. Li Y, Zhang X, Ge S, Gao J, Gong J, Lu M, Zhang Q, Cao Y, Wang DD, Lin PP, Shen L. Clinical significance of phenotyping and karyotyping of circulating tumor cells in patients with advanced gastric cancer. Oncotarget. 2014; 5:6594-602. https://doi.org/10.18632/oncotarget.2175.

6. Ge F, Zhang H, Wang DD, Li L, Lin PP. Enhanced detection and comprehensive in situ phenotypic characterization of circulating and disseminated heteroploid epithelial and glioma tumor cells. Oncotarget. 2015; 6:27049-64. https:// doi.org/10.18632/oncotarget.4819.

7. Yu M, Bardia A, Aceto N, Bersani F, Madden MW, Donaldson MC, Desai R, Zhu H, Comaills V, Zheng Z, Wittner BS, Stojanov P, Brachtel E, et al. Ex vivo culture of circulating breast tumor cells for individualized testing of drug susceptibility. Science. 2014; 345:216-220.

8. Cayrefourcq L, Mazard T, Joosse S, Solassol J, Ramos J, Assenat E, Schumacher U, Costes V, Maudelonde T, Pantel K, Alix-Panabières C. Establishment and characterization of a cell line from human circulating colon cancer cells. Cancer Res. 2015; 75:892-901.

9. Grillet F, Bayet E, Villeronce O, Zappia L, Lagerqvist EL, Lunke S, Charafe-Jauffret E, Pham K, Molck C, Rolland N, Bourgaux JF, Prudhomme M, Philippe C, et al. Circulating tumour cells from patients with colorectal cancer have cancer stem cell hallmarks in ex vivo culture. Gut. 2017; 66:1802-10.

10. Van de Ven AL, Tangutoori S, Baldwin P, Qiao J, Gharagouzloo C, Seitzer N, Clohessy JG, Makrigiorgos GM, Cormack R, Pandolfi PP, Sridhar S. Nanoformulation of Olaparib amplifies PARP inhibition and sensitizes PTEN/ TP53-deficient prostate cancer to radiation. Mol Cancer Ther. 2017; 16:1279-89.

11. Gewinner C, Wang ZC, Richardson A, Teruya-Feldstein J, Etemadmoghadam D, Bowtell D, Barretina J, Lin WM, Rameh L, Salmena L, Pandolfi PP, Cantley LC. Evidence that inositol polyphosphate 4-phosphatase type II is a tumor suppressor that inhibits PI3K signaling. Cancer Cell. 2009; $16: 115-125$. 
12. Fedele CG, Ooms LM, Ho M, Vieusseux J, O'Toole SA, Millar EK, Lopez-Knowles E, Sriratana A, Gurung R, Baglietto L, Giles GG, Bailey CG, Rasko JE, et al. Inositol polyphosphate 4-phosphatase II regulates PI3K/Akt signaling and is lost in human basallike breast cancers. Proc Natl Acad Sci USA. 2010; 107: 22231-22236.

13. Wan X, Zheng X, Pang X, Pang Z, Zhao J, Zhang Z, Jiang T, $\mathrm{Xu}$ W, Zhang Q, Jiang X. Lapatinib-loaded human serum albumin nanoparticles for the prevention and treatment of triple-negative breast cancer metastasis to the brain. Oncotarget. 2016; 7:34038-51. https://doi.org/10.18632/ oncotarget.8697.

14. Hernandez Maganhi S, Jensen P, Caracelli I, Zukerman Schpector J, Fröhling S, Friedman R. Palbociclib can overcome mutations in cyclin dependent kinase 6 that break hydrogen bonds between the drug and the protein. Protein Sci. 2017; 26:870-879.

15. Bockstaele L, Bisteau X, Paternot S, Roger PP. Differential regulation of cyclin-dependent kinase 4 (CDK4) and CDK6, evidence that CDK4 might not be activated by CDK7, and design of a CDK6 activating mutation. Mol Cell Biol. 2009; 29:4188-200.

16. Chen JF, Ho H, Lichterman J, Lu YT, Zhang Y, Garcia MA, Chen SF, Liang AJ, Hodara E, Zhau HE, Hou S, Ahmed RS, Luthringer DJ, et al. Subclassification of Prostate Cancer Circulating Tumor Cells by Nuclear Size Reveals Very Small Nuclear Circulating Tumor Cells in Patients With Visceral Metastases. Cancer. 2015; 121:3240-3251.

17. Lee JS, Melisko ME, Magbanua MJ, Kablanian AT, Scott JH, Rugo HS, Park JW. Detection of cerebrospinal fluid tumor cells and its clinical relevance in leptomeningeal metastasisof breast cancer. Breast Cancer Res Treat. 2015; 154:339-349.
18. Nayak L, Fleisher M, Gonzalez-Espinoza R, Lin O, Panageas K, Reiner A, Liu CM, Deangelis LM, Omuro A. Rare cell capture technology for the diagnosis of leptomeningeal metastasis in solid tumors. Neurology. 2013; 80:1598-1605.

19. Sahm F, Schrimpf D, Jones DT, Meyer J, Kratz A, Reuss D, Capper D, Koelsche C, Korshunov A, Wiestler B, Buchhalter I, Milde T, Selt F, et al. Next generation sequencing in routine brain tumor diagnostics enables an integrated diagnosis and identifies actionable targets. Acta Neuropathol. 2016; 131:903-910.

20. Mostert B, Sieuwerts AM, Kraan J, Bolt-de Vries J, van der Spoel P, van Galen A, Peeters DJ, Dirix LY, Seynaeve CM, Jager A, de Jongh FE, Hamberg P, Stouthard JM, et al. Gene expression profiles in circulating tumor cells to predict prognosis in metastatic breast cancer patients. Annals of Oncology. 2015; 26:510-516.

21. Khoo BL, Lee SC, Kumar P, Tan TZ, Warkiani ME, Ow SG, Nandi S, Lim CT, Thiery JP. Short-term expansion of breast circulating cancer cells predicts response to anticancer therapy. Oncotarget. 2015; 6:15578-93. https:// doi.org/10.18632/oncotarget.3903.

22. Lee GY, Kenny PA, Lee EH, Bissell MJ. Three-dimensional culture models of normal and malignant breast epithelial cells. Nat Methods. 2007; 4:359-365.

23. Serra V, Vivancos A, Puente XS, Felip E, Silberschmidt D, Caratù G, Parra JL, De Mattos-Arruda L, Grueso J, Hernández-Losa J, Arribas J, Prudkin L, Nuciforo P, et al. Clinical response to a lapatinib-based therapy for a Li-Fraumeni syndrome patient with a novel HER2V659E mutation. Cancer Discov. 2013; 3:1238-44. 